\title{
Silicon Nanowire Field-effect-transistor-based Biosensor for Biomedical Applications
}

\author{
Anran Gao, Shixing Chen, Yuelin Wang, and Tie $\mathrm{Li}^{* *}$ \\ Science and Technology on Micro-system Laboratory, Shanghai Institute of Microsystem \\ and Information Technology, Chinese Academy of Sciences, Shanghai 200050, China
}

(Received Novemver 30, 2017; accepted January 9, 2018)

Keywords: silicon nanowire, field-effect transistor, nanodevice, biosensor

The quantification and detection of biochemical species are of utmost importance for biomedical applications. Silicon nanowire field-effect transistors (SiNW-FETs) have recently attracted tremendous attention as a promising tool in biosensor design because of their ultrahigh sensitivity, selectivity, and label-free and real-time detection capabilities. Here, we review the device fabrication and biomedical applications of SiNW-FET sensors. CMOS-compatible approaches for SiNW fabrication are reported. The applications of SiNW-FETs in the fields of biomedical sciences are discussed. SiNW-FETs have been employed in the detections of, for example, $\mathrm{pH}, \mathrm{DNA}$, nucleic acid, proteins, and cancer biomarkers. The major limitations and future directions of SiNW-FET nanosensors are also discussed. In this paper, we clearly show the great potential of SiNW-FET devices as powerful new tools in biological and medical diagnostics.

\section{Introduction}

Because of the comparable sizes of biological macromolecules and nanomaterials [nanotubes, ${ }^{(1)}$ nanowires, ${ }^{(2)}$ and nanoparticles ${ }^{(3)}$, the integration of nanomaterials with biomolecules offers great potential for the development of sensing technologies of molecular-size scale or sensitive detection of biomolecules. ${ }^{(4)}$ The silicon nanowire field-effect transistor (SiNW-FET), ${ }^{(5-9)}$ one of the most promising platforms for unlabeled sensing, is emerging as a powerful sensor for recognizing a wide range of biological and chemical species with many attractive properties, including low cost, ultrahigh sensitivity, direct electrical readout, and multiplexed detection.

The binding of a charged species can result in the depletion or accumulation of carriers in the bulk of a nanowire relative to only the surface region of a planar device. The large surfaceto-volume ratio of SiNW causes its electrical properties, such as conductance, to be dominated by surface contributions. Therefore, the presence of charged species on the surface of an active nanowire induces a large fractional change in the nanowire conductance and enables relatively easy detection. A SiNW-FET device is connected to source (S) and drain (D) electrodes, and the conductance is switched by a gate $(\mathrm{G})$ electrode. For an n-type NW-FET device, when

\footnotetext{
*Corresponding author: e-mail: ylwang@mail.sim.ac.cn

** Corresponding author: e-mail: tli@mail.sim.ac.cn

http://dx.doi.org/10.18494/SAM.2018.1829
} 
negatively charged molecules (such as DNA) bind onto the SiNW surface, a depletion of carriers takes place in the NW, leading to a decrease in the current of the FET device. In contrast, the positively charged analytes would result in an accumulation of carriers, causing an increase in the conductance of the FET device.

In general, there are two major techniques for fabricating SiNWs: top-down ${ }^{(10)}$ and bottomup. $^{(11)}$ SiNWs prepared by the bottom-up approach are normally catalytically synthesized by chemical vapor deposition (CVD). This method is limited by complex integration owing to hybrid fabrication schemes that require transfer and low-yield assembly, as well as by the absence of reliable ohmic contacts. These largely hinder widespread application. Meanwhile, traditional top-down techniques ${ }^{(12)}$ such as e-beam lithography, which is popularly used to define nanostructures, also have troublesome barriers, such as expensive apparatus and serial processing to batch production.

Fundamentally different approaches for SiNW fabrication are reported in this work. The SiNWs were fabricated by a top-down method, allowing for mass production and compatibility with CMOS manufacturing technologies. The technique is cost-effective and suitable for the integration of complex nanowire sensor arrays in various possible applications. The fabricated SiNW-FETs have been employed in highly sensitive detections of, for example, $\mathrm{pH}$, DNA, proteins, and cancer biomarkers.

\section{Experimental Section}

\subsection{SiNW fabrication}

Nanowire fabrication was achieved on both (100) and (111) silicon-on-insulator (SOI) wafers. The nanowire fabrication started at a (100)-oriented separation by implantation of oxygen (SIMOX) SOI wafer (Fig. 1) was first thermally oxidized and etched by buffered HF (BHF) solution to reduce the top silicon layer thickness to $40 \mathrm{~nm}$. For microscale electrical leads, ion implantation of phosphorous adulterant, followed by $30 \mathrm{~min}$ of annealing in nitrogen at $1100{ }^{\circ} \mathrm{C}$, was carried out to form an effective contact region. By using tetra-methyl-ammonium hydroxide (TMAH; $25 \mathrm{wt} \%, 50{ }^{\circ} \mathrm{C}$ ) anisotropic silicon etching, the boundary of the top silicon layer was automatically aligned to the (110) direction and a very smooth (111) plane could be formed. Then a thin nitride film $(50 \mathrm{~nm})$ was deposited by low-pressure chemical vapor deposition (LPCVD) to protect the (111) plane previously defined. After the nitride film was patterned by ion-beam etching, the $100 \mathrm{~nm} \mathrm{SiO} 2$ layer beneath was totally removed using BHF. Then, using TMAH for the second time, we finally obtained controllable SiNW with a triangular cross section [Fig. $1(\mathrm{~h})]$.

The fabrication of an SiNW array can also start from an n-type (111)-oriented SOI wafer, as shown in Fig. 2. A highly controllable size-reduction technology based on the unique self-stop etching planes for a (111) wafer with shallow wet-etched cavities on the wafer was developed. The key process for SiNW fabrication is the formation of a highly down-scaled silicon wall structure, which is used to generate the SiNW via self-limiting oxidation. The width of the silicon wall can be precisely controlled using three controllable process parameters: dry-etching 


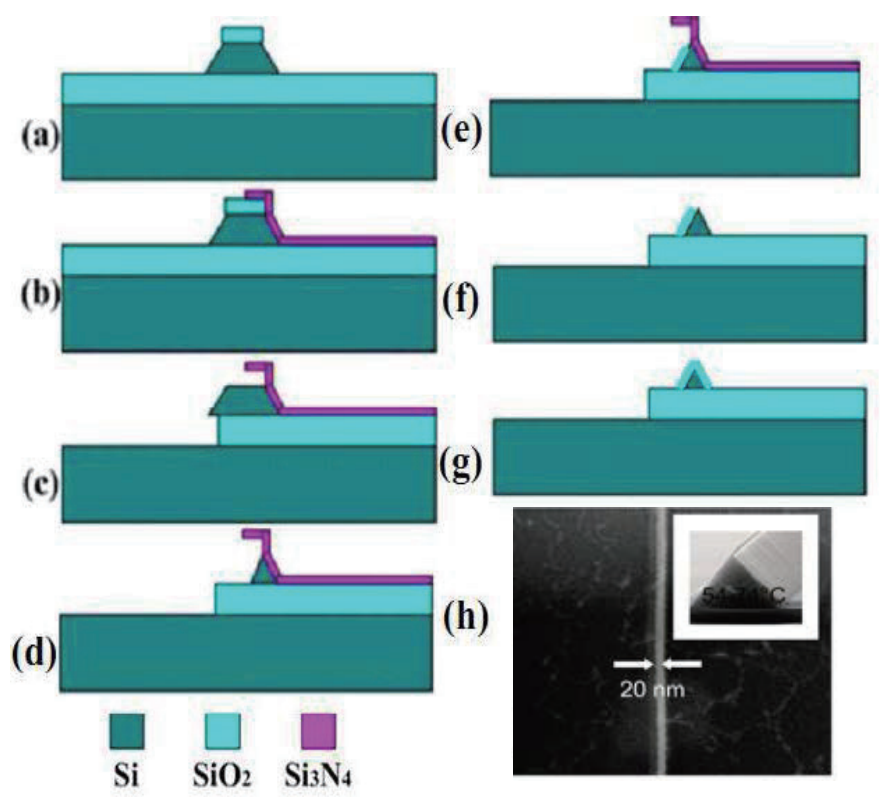

Fig. 1. (Color online) Top-down process for SiNW fabrication by an anisotropic wet etching approach. Reprinted with permission from Ref. 10.

(a)

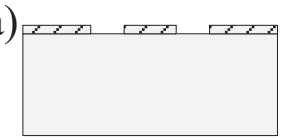

(b)

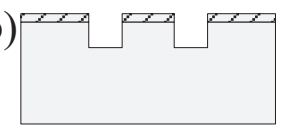

(c)
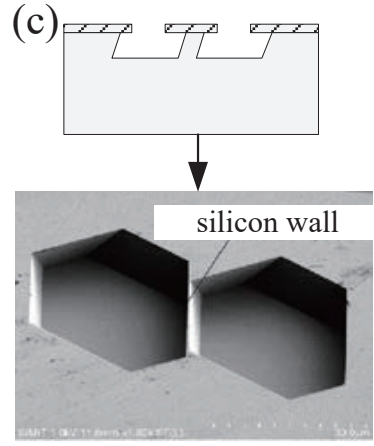

7 (d)

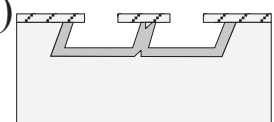

(e)

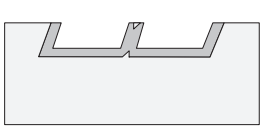

(f)
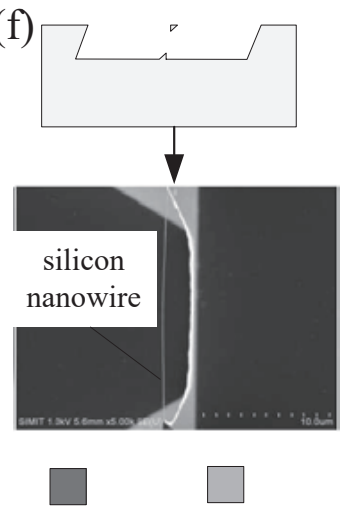

Fig. 2. Fabrication of SiNW from an n-type (111)-oriented SOI wafer by self-limiting oxidation.

depth $\tau$, offset angle $\theta$, and wet-etching time. Firstly, a 100-nm-thick silicon nitride layer was covered and patterned. Then, the silicon underneath was dry-etched to a depth of $\sim 2.5 \mu \mathrm{m}$. After anisotropic wet etching in potassium hydroxide $(\mathrm{KOH})$ solution, cavities were formed. 
A $\mathrm{SiO}_{2}$ layer of $\sim 500 \mathrm{~nm}$ thickness was then thermally oxidized on the bottoms and sidewalls of the wet-etched cavities. A suspended SiNW with an inverted-triangle cross section was finally generated after the removal of the oxide. The developed top-down techniques facilitate compatibility with the CMOS surface manufacturing process without positioning or contact problems.

\subsection{SiNW surface modification}

For SiNW surface functionalization, the SiNW was first cleaned with oxygen plasma in order to clean the sample surfaces as well as to generate more hydrophilic surfaces with hydroxyl terminating the silicon oxide surface. Then, it was functionalized by exposing the surface to (3-aminopropyl)triethoxysilane (APTES) solution (2\% ethanol) overnight, followed by rinsing with absolute ethanol to remove unreacted APTES. After that, a self-assembled monolayer with a terminal amino group was prepared by blowing the surface with nitrogen, followed by heating at $120{ }^{\circ} \mathrm{C}$ for 5 min. A biofunctional linker, glutaraldehyde, was then covalently attached onto the amine-terminated surface by immersing the chip in a solution of $2.5 \%$ glutaraldehyde in buffer solution $(20 \mathrm{mM} \mathrm{NaCl}, 10 \mathrm{mM}$ phosphate, $\mathrm{pH} 7.4)$ at $37^{\circ} \mathrm{C}$ for 1

h. Finally, the probes for target molecules were attached on the SiNW surface in buffer solution followed by thorough washing with the same buffer.

\section{Application of SiNW-FET Biosensor}

\subsection{Ion detection}

The first example of SiNWs as ions transducers was reported by Lieber's group in 2001. ${ }^{(11)}$ The back-gate-controlled field effect on device sensitivity was studied using a $\mathrm{pH}$ sensing experiment by our group. ${ }^{(10)}$ Figure $3($ a) shows as-prepared SiNW devices characterized as nanoscale $\mathrm{pH}$ sensors. The real-time current of the silicon nanowire decreased stepwise with a discrete change in $\mathrm{pH}$ from 5.0 to 9.0 [Fig. 3(c)]. Furthermore, the change in current was also reversible when the solution $\mathrm{pH}$ was reversed. The current from 5.0 to 9.0 showed nonlinear $\mathrm{pH}$ dependence [Fig. 3(e)], which is consistent with the $\mathrm{pH}$-dependent surface charge density derived from silica.

SiNWs were then silanized at the exposed, dominant Si (111) planes with APTES. The terminal amine functions as a proton receptor [Fig. 3(b)] that can undergo protonation and deprotonation with $\mathrm{pH}$ variation, and the change in surface charge can chemically gate the SiNW-FETs. The plot of current versus $\mathrm{pH}$ showed that the $\mathrm{pH}$ dependence became linear over the $\mathrm{pH}$ range of 7.0 to 9.0 [Fig. 3(f)]. At low $\mathrm{pH}$, the amino group was protonated and acted as a positive gate that accumulates electron carriers in the n-type SiNW, thus increasing the conductance. This behavior clearly demonstrated that the sensing mechanism was indeed the result of a field effect, analogous to applying a voltage using a physical gate electrode. 

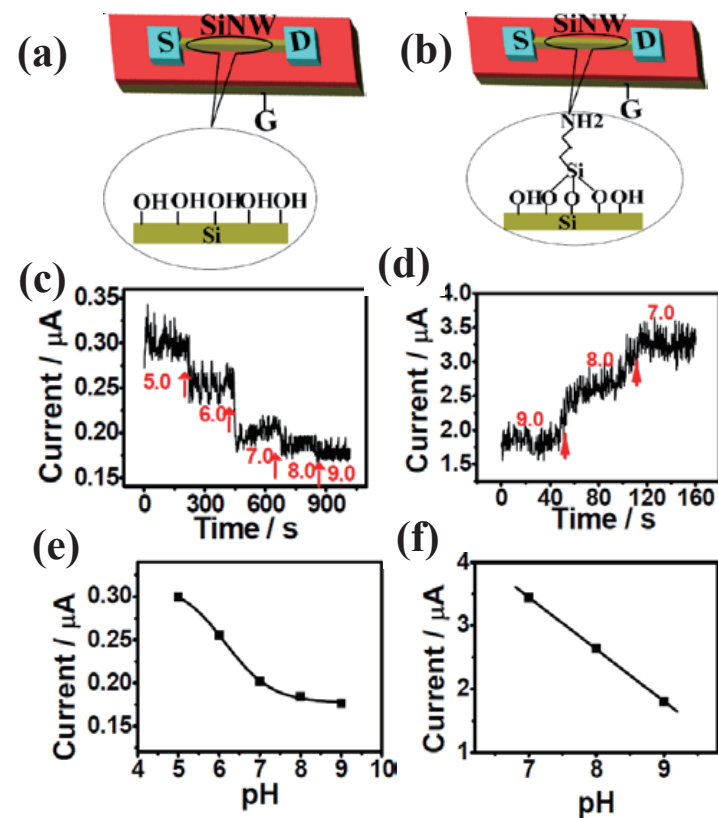

(d)
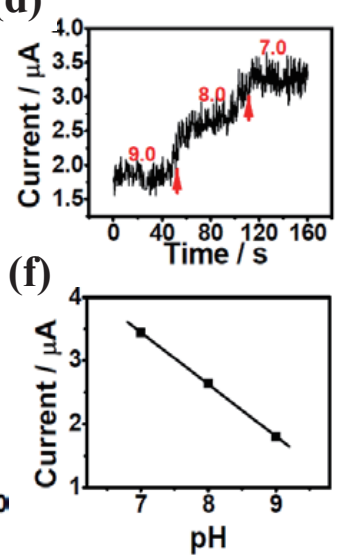

Fig. 3. (Color online) Schematic illustrations of (a) unmodified SiNW FET and (b) APTES-modified SiNW. Responses from (c) unmodified and (d) APTES-modified SiNW devices to solutions of different pH values. Plots of the current of (e) unmodified and (f) APTES-modified SiNW devices as a function of pH. Reprinted with permission from Ref. 10.

\subsection{Nucleic acid detection}

We employed the n-type SiNW-FET device to electrically detect DNA hybridization (Fig. 4). Capture probe DNA with a terminal carboxyl group was conjugated to the amine of APTES-modified SiNWs, with the help of $N$-hydroxysuccinimide and 1-ethyl-3-(3dimethylaminopropyl) carbodiimide. When the buffer solution flowed through the sensor surface, the electrical response of the SiNW-FET remained nearly unchanged. Significantly, when a $1 \mathrm{nM}$ solution of fully complementary target DNA was introduced, we observed a rapid decrease in the electrical current to nearly half of the initial value within seconds. Note that the hybridization process could be monitored in real time with this SiNW-FET sensor. The decrease in electrical current suggested that the binding of negatively charged target DNA to the gate dielectric of the n-type SiNW-FET results in the depletion of carriers. As a control experiment, target DNA of the same concentration was passed through an unmodified SiNWFET but did not lead to significant current change, suggesting the absence of the nonspecific binding of target DNA to the SiNW surface.

Further experimentation indicates that this SiNW-FET nanosensor revealed ultrahigh sensitivity for the rapid and reliable detection of $1 \mathrm{fM}$ target DNA and high-specificity singlenucleotide polymorphism discrimination. As a proof-of-concept for multiplex detection with this small, mass-producible sensor array, we demonstrated the simultaneous selective detection of two pathogenic strains of virus DNA sequences (H1N1 and H5N1) of avian influenza. 


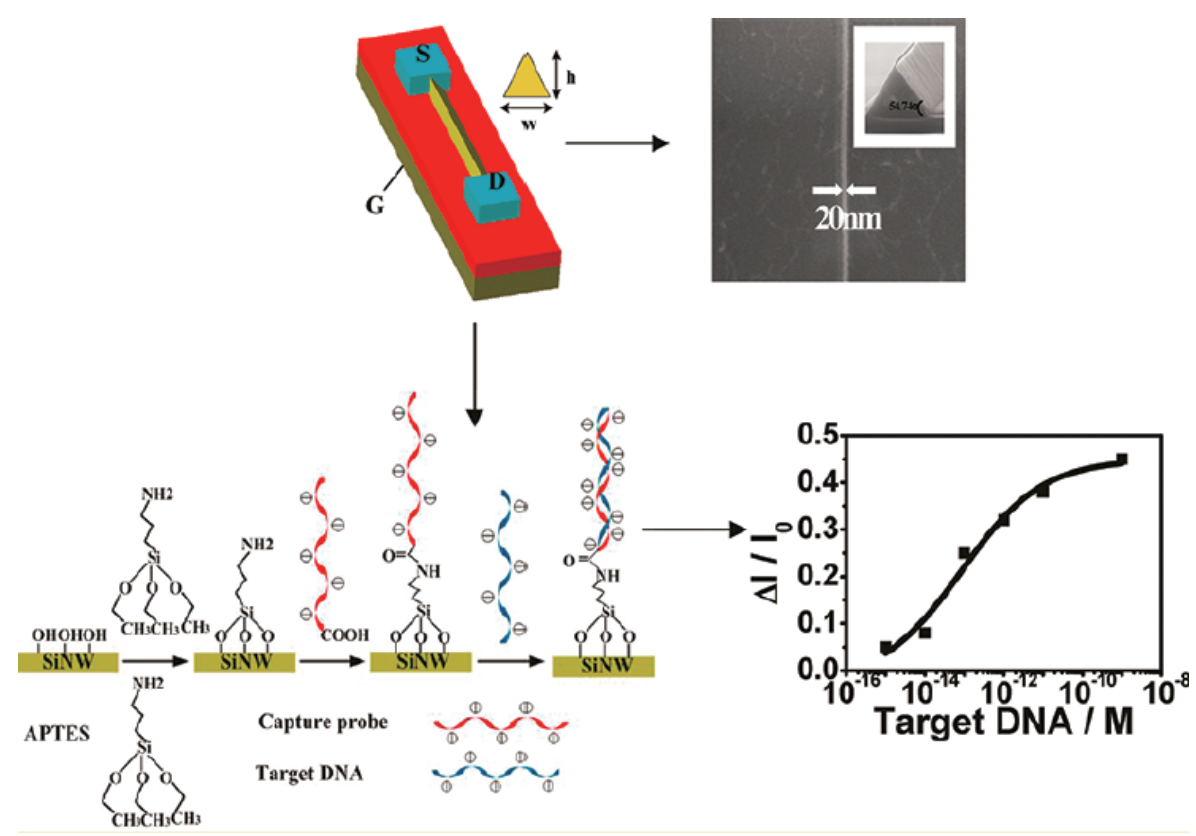

Fig. 4. (Color online) Scheme for the fabrication of SiNW-FET biosensor for highly sensitive DNA detection. Reprinted with permission from Ref. 10.

\subsection{Protein detection}

The sensitive and quantitative analysis of proteins is central to disease diagnosis, drug screening, and proteomic studies. In this study, silicon nanowire biosensors for the detection of prostate-specific antigen (PSA) biomarkers ${ }^{(13)}$ were realized by immobilizing specific antibodies onto a SiNW surface [Fig. 5(a)]. For one nanowire in the array, when the buffer solution (0.01×) flowed through the anti-PSA-modified SiNW sensor surface, the electrical response of the SiNW-FET remained stable. Significantly, when the solution containing $1 \mathrm{pg} / \mathrm{ml}$ PSA was introduced, the time-dependent current exhibited a rapid increase. Notably, the current remained nearly unchanged after washing with buffer solution without PSA, indicating that the electrical current change is caused by the specific binding events and there is little nonspecific bonding.

The sensitivity of the SiNW-FET sensor was investigated by testing it with a series of concentrations of PSA. The real-time response of current upon the injection of various concentrations of PSA is illustrated in Fig. 5(b). The sparseness of nonspecific binding and adsorption of other molecules was also demonstrated with a series of concentrations of BSA. The SiNW response as a function of PSA and BSA concentrations is shown in Fig. 5(c). The electrical current change increased monotonically with the logarithm of PSA concentration, whereas the current remained nearly unchanged with the logarithm of BSA concentration. 
(a)

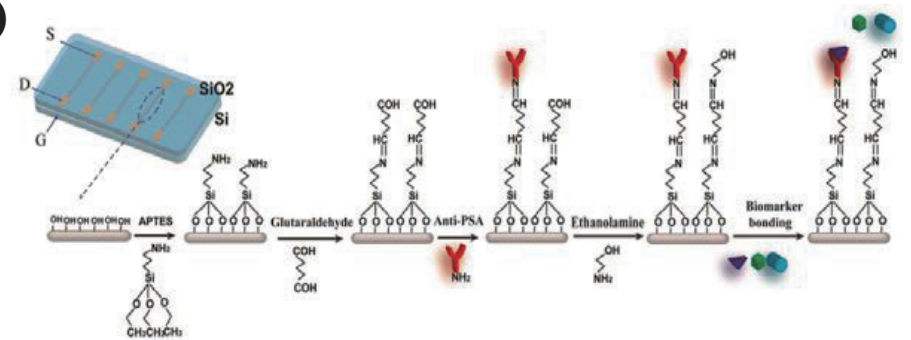

(b)

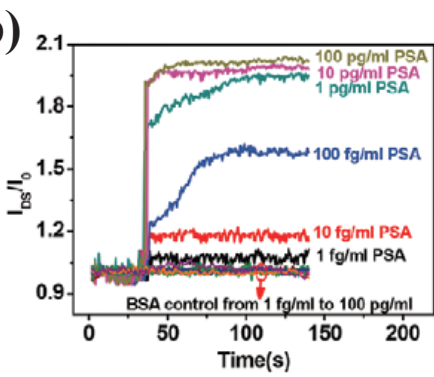

(c)

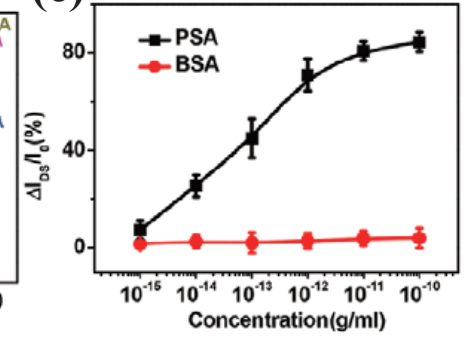

Fig. 5. (Color online) (a) Schematic illustration of the chemical process for real-time and label-free electrical detection of PSA. (b) Plots of normalized current change versus time with PSA at a series of concentrations for the anti-PSA-modified SiNW device. (c) Normalized current change as a function of the logarithm of PSA concentration. Reprinted with permission from Ref. 13.

\subsection{Disease diagnostics}

Cancer is often silent in its early course and diagnosed at an advanced stage, at which time treatment outcomes are not favorable. With the development of diagnostic methods, patients can be diagnosed by analyzing tumor biomarkers released by cancer cells or created by the body. They are providing great opportunities for enhancing the efficiency of early stage cancer detection in recent years. Specifically, carcino embryonic antigen (CEA) has been suggested to be a valuable biomarker for cancer diagnosis at an early stage. Recent findings also show that microRNAs (miRNAs) stably expressed in the human bloodstream hold considerable promise for early cancer detection.

We chose CEA and miRNA-126 as two typical cancer biomarkers; they have been recognized as characteristic predicators in the early screening and clinical diagnosis of lung cancer. ${ }^{(14)}$ In this work, we have also created a fluid-exchange system using polydimethylsiloxane (PDMS) with microfluidic chambers that can rapidly transport a sufficient number of molecules to the SiNW surface to enable sensing. The schematic design of label-free multiplexed biomarker detection is shown in Fig. 6(a). The capture probe DNA and anti-CEA were covalently immobilized on SiNW surfaces separately in microchambers. The SiNW response as a function of miRNA and CEA concentrations is shown in Figs. 6(b) and 6(c), respectively. The electrical current change increased monotonically with the logarithm of target molecule concentration The nanosensor could reliably detect miRNA and CEA down to concentrations as low as $0.1 \mathrm{fM}$ and $1 \mathrm{fg} / \mathrm{ml}$, respectively. 

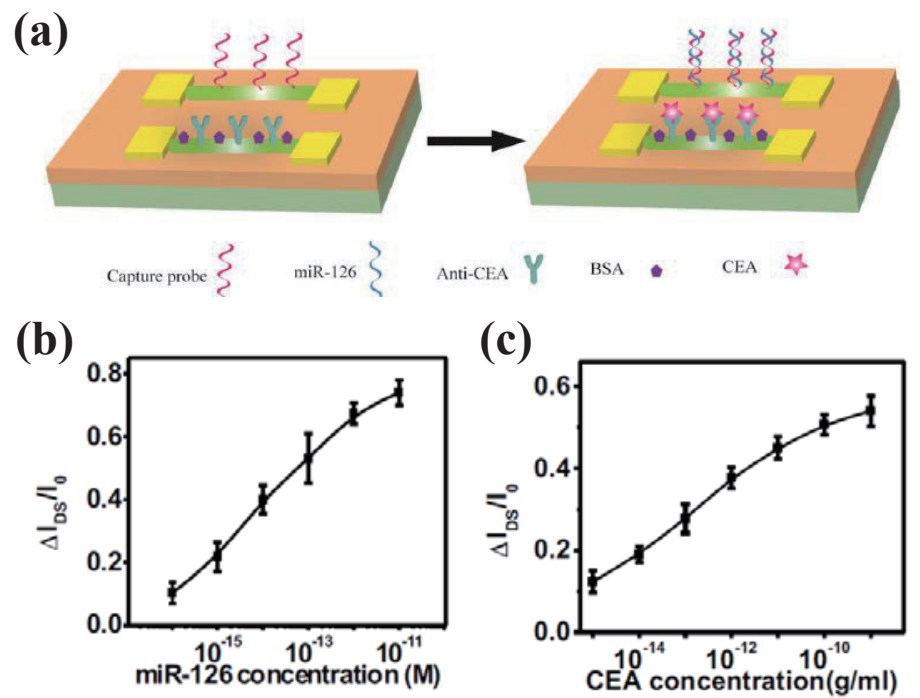

Fig. 6. (Color online) (a) Schematic illustration of label-free multiplexed electrical detection of lung cancer biomarkers CEA and miRNA-126. Relative current change of SiNW nanosensor as a function of the logarithm of target (b) miRNA and (c) CEA concentrations. Reprinted with permission from Ref. 14.

\section{Conclusions}

SiNW-FET-based biosensors are attractive for the direct, label-free, real-time, and sensitive detection of target analytes with ultralow detection limits. We developed a CMOS-compatible anisotropic wet etching technique for the fabrication of SiNW arrays and a high-performance SiNW-FET device. This device allows electrical sensing in real time, and multiplexing is possible owing to the presence of 16 individually aligned SiNWs. We have demonstrated that this nanosensor can rapidly and sensitively detect various targets, including $\mathrm{pH}$, DNA, microRNA (miRNA), proteins, and cancer biomarkers. Given the extraordinary ability of real-time label-free biomolecular detection and the small size and low cost of mass-producible CMOScompatible devices, we expect that the SiNW-FET biosensor will provide a generic platform for numerous applications including point-of-care test. In the coming years, the study of the SiNWFET sensing technique will be focused on how to advance this nanosensor to a commercial product. We believe that SiNW-FETs will play a significant role in the development of biomedical sensors using nanotechnology in the future.

\section{Acknowledgments}

We thank the National Key Research and Development Program of China (Nos. 2017YFB0405403 and 2017YFA0207103), Project of National Natural Science Foundation of China (Nos. 91323304, 81402468, 61327811, and 91623106), Project for Shanghai Outstanding Academic Leaders (No. 15XD1504300), and Youth Innovation Promotion Association, CAS, for the financial support. 


\section{References}

1 D. S. Engstrom, V. Savu, X. N. Zhu, I. Y. Y. Bu, W. I. Milne, J. Brugger, and P. Boggild: Nano Lett. 11 (2011) 1568.

2 A. K. Wanekaya, W. Chen, N. V. Myung, and A. Mulchandani: Electroanalysis 18 (2006) 533.

3 N. C. Tansil and Z. Q. Gao: Nano Today 1 (2006) 28.

4 M. A. Fraga, R. S. Pessoa, D. C. Barbosa, and V. J. T. Airoldi: Sens. Mater. 29 (2017) 39.

5 K. I. Chen, B. R. Li, and Y. T. Chen: Nano Today 6 (2011) 131.

6 Z. Li, B. Rajendran, T. I. Kamins, X. Li, Y. Chen, and R. S. Williams: Appl. Phys. A 80 (2005) 1257.

7 N. Lu, P. F. Dai, A. R. Gao, J. Valiaho, P. Kallio, Y. L. Wang, and T. Li: ACS Appl. Mater. Interfaces 6 (2014) 20378.

8 N. Lu, A. R. Gao, P. F. Dai, S. P. Song, C. H. Fan, Y. L. Wang, and T. Li: Small 10 (2014) 2022.

9 E. Stern, J. F. Klemic, D. A. Routenberg, P. N. Wyrembak, D. B. Turner-Evans, A. D. Hamilton, D. A. LaVan, T. M. Fahmy, and M. A. Reed: Nature 445 (2007) 519.

10 A. R. Gao, N. Lu, P. F. Dai, T. Li, H. Pei, X. L. Gao, Y. B. Gong, Y. L. Wang, and C. H. Fan: Nano Lett. 11 (2011) 3974.

11 Y. Cui, Q. Q. Wei, H. K. Park, and C. M. Lieber: Science 293 (2001) 1289.

12 K. Bedner, V. A. Guzenko, A. Tarasov, M. Wipf, R. L. Stoop, D. Just, S. Rigante, W. Y. Fu, R. A. Minamisawa, C. David, M. Calame, J. Gobrecht, and C. Schonenberger: Sens. Mater. 25 (2013) 567.

13 A. R. Gao, N. Lu, P. F. Dai, C. H. Fan, Y. L. Wang, and T. Li: Nanoscale 6 (2014) 13036.

14 A. R. Gao, X. Yang, J. Tong, L. Zhou, Y. L. Wang, J. L. Zhao, H. J. Mao, and T. Li: Biosens. Bioelectron. 91 (2017) 482.

\section{About the Authors}

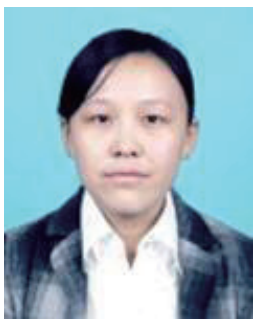

Anran Gao received her B.S. degree from Qingdao University in 2008 and her Ph.D. degree from Shanghai Institute of Microsystem and Information Technology, Chinese Academy of Sciences, in 2013. Her research interests include design, analysis, and fabrication of semiconductor devices and their applications in biochemical sensing.

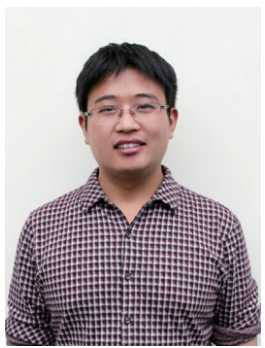

Shixing Chen is currently an assistant Research Fellow at Shanghai Institute of Microsystem and Information Technology, Chinese Academy of Sciences. He received his Ph.D. degree from Shanghai Institute of Applied Physics, Chinese Academy of Sciences. His scientific interests focus on nanomaterials for electrochemical and bioanalytical applications and biosensor construction.

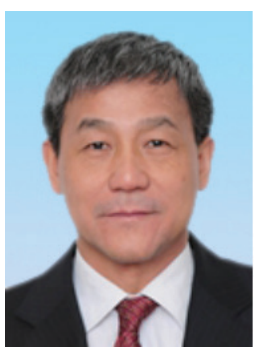

Yuelin Wang received his B.S. degree from Zhejiang University in 1982, his M.S. degree from Harbin Institute of Technology in 1985, and Ph.D. degree from Tsinghua University in 1989. He is currently a professor at Shanghai Institute of Microsystem and Information Technology. His research interests include microelectromechanical and nanoelectromechanical systems. 


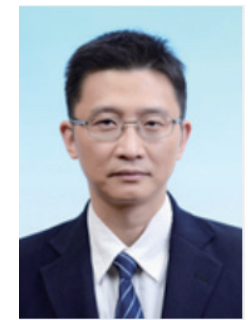

Tie Li received his B.S. and Ph.D. degrees in condensed matter physics from the University of Science and Technology of China, Hefei, in 1992 and 1997, respectively. He is currently a professor in the MEMS Lab at Shanghai Institute of Microsystem and Information Technology. His research interests include the mechanism, fabrication, and application of micro-nano sensors. 